\title{
$\angle$ Research Square \\ Efficient Cloud-Based Resource Sharing Through Multi-Tenancy and Load Balancing: An Exploration of Higher Education and Digital Libraries
}

Saeed Q. Al-Khalidi Al-Maliki ( $\square$ salkhalidi@kku.edu.sa )

King Khalid University https://orcid.org/0000-0001-9570-0031

\section{Research Article}

Keywords: Cloud storage, private cloud, public cloud, multi-tenant, single-tenant, load balancing, digital data

Posted Date: May 2nd, 2022

DOI: https://doi.org/10.21203/rs.3.rs-1311794/v3

License: (c) (i) This work is licensed under a Creative Commons Attribution 4.0 International License.

Read Full License 


\section{Abstract}

Cloud computing has gained considerable attention in academia and industry in recent years. The cloud facilitates data sharing and enables cost efficiency, thus playing a vital role today as well as for the foreseeable future. In this paper, we briefly discuss the application of multi-tenant and load-balancing technologies to cloud-based digital resource sharing suitable for academic and digital libraries. As a new paradigm for digital resource sharing, we propose improving the current user service model with private cloud storage for other sectors, including the medical and financial fields. This paper gives a summary of cloud computing and its possible applications, combined with digital data optimisation for web-based services. This study could aid in the identification and generation of cloud-based services that use multitenant and load-balancing methodologies for the best quality of service.

\section{Introduction}

Based on user demand, cloud computing acts as a resource-sharing tool for accessing infinite computing services and resources. Cloud computing has many advantages, such as reducing usage time and flexible computing capabilities [1]. It appears to provide limitless storage as well as the capacity to swiftly share and process data [2]. Cloud computing technology for university libraries and, in particular, digital libraries could soon pave the way for new services. Digital library services will become more professional and effective, resulting in increased knowledge benefits for university libraries [3]. University libraries must constantly improve by implementing new information technologies (IT), such as cloud computing, to deliver services to staff and students. University libraries could soon be building and managing their own data centres to maintain more control over applications and data stores containing sensitive and private information [4].

A digital library is a development-orientated software and hardware integration platform established through product and technical integration. With the popularisation of digital library technology, it has become possible to provide high-quality information services. Cloud computing can provide an effective solution in this regard [5].

The multi-tenant application is a perfect choice for any environment that wants to start with fewer hardware requirements and easier onboarding. Additionally, it is ideal for users who do not have the internal resources to deal with the maintenance requirements of single-tenant software as a service (SaaS) environments [6]. A functional diagram of multi-tenant and single-tenant modules is shown in Figure 1. Cloud structures store, manage and govern data based on user requirements. Hence, designing and implementing flexible architecture by using multi-tenant facilities provides a significant level of service.

Multi-tenancy refers to the ability to run multiple users of an application on a shared infrastructure. Our main goal is to reduce the cost per user over a dedicated system where each user has their own 
environment by using multi-tenancy. The cloud user will only be able to see their own data with this feature. It will create a cloud of the activities of one set of users that cannot affect another set.

Automation of deployment, scaling and operations of application containers across clusters of hosts will be possible with it.

The remainder of this research article is divided into the following sections: Section 2, the literature review; Section 3, the significance of the research; Section 4, the proposed architecture design; Section 5, the load-balancing model; Section 6, the implementation structure of the proposed scheme; Section 7, the simulation result and analysis; Section 8, the benefits of the proposed structure; Section 9, the limitations of multi-tenant resource sharing; and finally, Section 10, the conclusion.

\section{Literature Review}

This study presents numerous research articles related to cloud adoption studies on digital resource sharing. A study by Aljerewi (2018) on the effects of cloud computing on the cognitive skills of students enrolled in the curriculum of teaching techniques at the Faculty of Education at Princess Nourah Bint Abdul Rahman University in Riyadh showed that using cloud computing applications improved the students' knowledge as well as the rate of project completion [7].

To understand the functioning of the cloud library, there is a need to understand cloud computing. Before advancing the use of cloud technology in an organisation, the management must assess the advantages, readiness of team members and the results it will bring to stakeholders [8]. Cloud computing produces a resource access model that depends entirely on an internet connection and provides a platform to both data owners and users. It allows data owners to store data in the servers and regulates user access to the information. Information security relies on the access policies of the service provider, and the direction and storage policies dictated by the owners.

There are four National Institute for Standards and Technology (NIST)-recognised service models offered by cloud service providers (CSPs) [9]. SaaS is a licensed version of the software provided by the CSP, and payment is made according to provider specification [10]. Infrastructure as a service (laaS) provides resources, such as storage, safety software and network, through the CSP [11]. laaS is the foundation of cloud computing since it establishes the resources required by CSPs to provide hosting services to data owners and access to users [12]. Platform as a service (PaaS) bridges the gap between laaS and SaaS; users can create, run and deploy applications in the cloud environment [11]. PaaS allows data owners to store data in the cloud servers and control access by users.

Cloud library applications use a cloud service model. Educational materials are stored in the cloud server, which is accessed through the online application. Publishers and researchers own the content and pay for storage in the cloud server. The owners restrict access, both free and commercial. The access, security 
and safety of the material in the cloud library server depend on the policies and control of the access software and platform.

Cloud computing is deployed in four NIST-approved models: community, public, private and hybrid [13]. A community model integrates groups of users with the same interest in a particular cloud platform. A public model provides deployment of a resource that is transparent to users. A private deployment model is developed for and dedicated to a specific user and controls access for that user. A hybrid model combines these models of access and deployment. A library can apply the model it requires for library management based on the advantages and control systems.

Determining the most efficient means of securing data within the Hadoop cloud environment is HCX's goal. CSPs provide the necessary virtualised resources to run cloud services and implement our flexible research system (FRS) initiative. The large scope, which could be potentially realised through a cloud environment, brings security, privacy, financial and occupancy questions into the fold. A highly innovative tool for accomplishing such a feat is Hadoop [14]. Hadoop is an open-source implementation of the program Google MapReduce, which allows the cloud architect to handle large amounts of data, while also utilising the initial properties of Google MapReduce [12]. This can be helpful for our FRS software, as, thanks to Hadoop technology, a region of clients can be processed at a much speedier rate. Unfortunately, Hadoop can only solve volume problems, not security issues. Dealing with such a high influx of data does not allow users to set security measures or secure access methods on incoming data. It is important to specify why Hadoop is advantageous and what constitutes big data. The big data factors include volume, variety, velocity, variability and complexity. Volume comprises transaction data storage, live streaming data and data collected from sensors. Volume cannot be achieved without a framework equipped to speedily filter through candidates. It is important to note the important roles played by the other four factors. Variety refers to the several different formats of the data, such as text documents and video or audio. Velocity refers to the speed at which data originates and the time frame for processing the data. Variability refers to the inconsistent flow at which data may be received, and complexity is based on the source from which data originates.

Before processing can begin, data must be matched, linked, cleansed and converted into manageable formats. Several organisations use big data but have not invested in the necessary precautions to protect volatile data from harm. Security attacks on a vast amount of information could mean complete financial ruin and loss of integrity if the data is not secured. Some necessary actions to take regarding big data include the classification of information through encryption, honeypot detection and logging. A proven method for securing big data is fraud detection. The only valuable method for handling intrusion threats against organisations prioritising big data is a big data-styled analysis. Techniques explained earlier in this section are specifically dedicated to identifying threats early, reporting them to authorised administrators and handling them promptly.

Though transitioning into a cloud environment is advantageous, several issues plague the process. The interconnection of several different systems, including virtualisation, operating systems, networks and 
memory management, must all be accounted for when employing security measures for a cloud environment. This is especially true for the virtual and physical elements of devices within the environment; these matters can be potentially harmful for an organisation if handled poorly. Encryption is essential within a cloud environment and in the process of data sharing [15]. Due to the variability big data presents, it is necessary to institute many solutions in creating a secure cloud environment. These include file encryption and network encryption. Disorganised data in hardware is vulnerable to cyberattacks. To avoid this, data must be encrypted by maintaining specific encryption keys for various devices, with critical data being locked away within impenetrable firewalls [12]. Network encryption details the remote procedure call (RPC) method of encrypting transmission within a network to prevent attackers from modifying packets as they see fit. Administrators are also encouraged to deploy honeypot nodes to lure cyber attackers within the cloud environment to be caught and traced back to their source. Nodes should be monitored, as attackers can quickly deploy harmful nodes within the environment that may go undetected in the absence of authentication of every node within a cluster. Practical authentication methods like Kerberos can aid in keeping malicious nodes out of the cloud environment.

\section{Significance Of The Research}

An educational institution is dedicated to maintaining and improving its capacity to preserve and safeguard paper-based documents, especially its rare, distinctive and otherwise valuable papers. There is a significant public and private educational need to establish robust digital storage media for exchanging and sharing valuable information, including electronic books, medieval manuscripts, atlases, early prints, maps, literary manuscripts and additional paper objects within educational institutions. This is very difficult to maintain for most institutions, and using library materials can be difficult for the user. Cloud architecture can store a large pool of data for future reference and development [16], [17].

The current cloud computing industry does not have specific development standards, and a user has to depend on the vendors' specifications [15]. NIST provides guidelines on how cloud computing platforms should operate. Cloud services are guided by six principles, provided as guidance by the Cloud Security Alliance [13]. These include abstraction of infrastructure, consumption, the allocation utility model, democratisation of resources, service-oriented architecture and dynamism.

The vast resources at both physical and virtual libraries are challenging to manage using simple mechanical methods. The introduction of cloud computing for libraries has made management much more accessible through the applications provided. According to Tyagi et al. (2015), cloud libraries provide a centralised method of storing resources and allow simultaneous access to different users at any time and at any location [11]. Different applications and services are provided in cloud libraries, such as automated libraries, searching services, digital libraries, file storage and community resource centres.

\section{Proposed Architecture Design}


The proposed architecture model is designed for cloud libraries and is suitable for any resource-sharing centre with a private cloud server. Application-level software, which is available on the market for a low price, can help with data storage. The application-level software is an internet-enabled device that will link to the library's own external hard drives, allowing anyone with access permissions to access the data on the private cloud server.

The proposed architecture is shown in Figure 2, which is intended to demonstrate how data should be searched and retrieved from a cloud storage system. This architectural design is based on the private cloud computing service model. The private cloud storage holds the library's data, including different types of documents, PDF files, journals and electronic books. All these items of data are assigned a unique identification code for easy access.

\section{Load-balancing Method}

Implementing the load-balancing structure distributes traffic across the data distribution links. Popular and well-known methods of load balancing include round-robin, Least, observed method and fastest connections. A data distribution approach in which a single instance of software operates on a server and supports several users is known as multi-tenant. Table 1 describes the significant benefits of the multi-tenant approach over the single-tenant one. The multi-tenant approach dynamically shares access to system resources based on user requirements.

If proper security requirements and engineering methodology are not well-implemented in a system, it can result in a cyberattack. Well-implemented security requirements lower this risk, reduce costs and protect digital business assets. One technique for implementing security requirements, developed by Carnegie Mellon University, is system quality requirement engineering (SQUARE) [18].

The SQUARE methodology is a nine-step process to ensure the security and privacy of IT systems and applications in the early stages of a product lifecycle. It is focused on building security and quality concepts and can also be used for documenting and analysing the security and quality aspects of a development project. The nine-step process helps organisations build security and privacy into the early stages of the product lifecycle.

This model is also used for documenting and analysing security aspects of fielded systems that use machine learning (ML). Unlike Touch ID, a critical component of $M L$ is the ability to learn changes in facial features over time. More importantly, due to the high accuracy of the integration process, ML can easily detect false information. Because FRS is completely automated, it eliminates time fraud. Over time, the system can potentially face security risk challenges, such as hacking; future improvements and system modifications can help eliminate the security risks. Using SQUARE can enable an organisation to develop secure software system programs that are cost-effective and efficient. 
Table 1. Difference between single tenancy and multi-tenancy

\begin{tabular}{ccc}
\hline Feature & Single Tenancy & Multi-Tenancy \\
\hline Price & Expensive & Cost-effective \\
\hline Adding custom features & Easy & Complex \\
\hline Security & Secure by design & Depends on the vendor \\
\hline Performance & Depends on the database module & API reduces workload \\
\hline Maintenance & Depends on vendor & Depends on the service provider \\
\hline Backup and recovery & Easy & Complex \\
\hline Host migration & Flexible & Moderate \\
\hline Updates and patches & Depends on individual customer & Automatic database upgrade \\
\hline
\end{tabular}

\section{Implementation Structure}

Figure 3 shows a single instance of a software application service that serves multiple users and has multiple-tenancy architecture, where each user is known as a tenant. To implement this architecture, there are multiple strategies, extending from highly isolated to everything shared with the user. Multi-tenant architecture is found in private, public and educational environments and enables the data of each tenant to be separated from that of others. The proposed architecture model allows all tenants to use a logical and physical database environment. All their data is securely separated within the same database by implementing unique identifiers for each user.

In general, multi-tenant design focuses on the components of the application, infrastructure and network [20].

\subsection{Application}

A multi-tenant app is a server app serving multiple users on a single code. It depends on a PaaS or SaaS development environment that supports scaling and configuration of multi-tenancy.

\subsection{Infrastructure}

Infrastructure is hardware that enables multi-tenant supporting software. Deficient infrastructure may negatively affect loading time, speed, data storage and transmission, backup protocols, backup plans and recoveries.

\subsection{Network}

The network is a platform; it provides security for a multi-tenant provider. On the open network medium, maximum violations occur when the data flow between users is not encrypted. Secure Socket Layer is responsible for the flow of data and encryption/decryption techniques.

\section{Simulation And Result Analysis}

The client phase helps students order printed books, literary manuscripts, maps and atlases, prints and all other paper objects within the educational institution, while the server phase illustrates the library nodes that execute the load-balancing algorithm in sequence. When a student orders a book or document, the 
order will initially be assigned to a cloud library node for that particular book or document for extraction or retrieval without the need to wait in a queue. To verify the load-balancing performance and multi-tenant technique, we have used the GNS3 simulator to identify the data downloading stream with 10 nodes, known as client systems, connected to the cloud database server (Figure 3 ). The resulting performance of the 10 clients and five jobs per client-activated system with the server is shown in Table 2. Our ultimate goal for the proposed multi-tenant load-balancing technique is to improve/understand how it delivers digital data to the client/student/customer. Table 2 shows the least number of active jobs at the time of the client system arrival rate $(\lambda$ or $t)$ and completion rate $(\mu)$. We have used a network simulator tool to design multi-tenant and load-balancing techniques, leading to working and cost efficiencies.

For the simulation, node $=10$, and the number of service facilities in the system, $\mathrm{k}=5$ for $\mathrm{M} / \mathrm{M} / \mathrm{C}$. Table 2 shows the results of our single-tenant and multi-tenant system simulations. The goal is to show how using a multi-tenant system is more efficient and time-saving than using a single-tenant system by comparing the throughput of each system. The number of completed requests per unit of time is referred to as throughput, while delay is the time taken to execute a request in the queue. In our simulation, randomly picked values are used to adapt to the pattern in which a request for resources enters a given system.

Table 2. Simulation table

\begin{tabular}{cccccc}
\hline 口 & $\boldsymbol{\mu}$ & \multicolumn{2}{c}{ Throughput (t) } & \multicolumn{2}{c}{ Delay (tq) } \\
\cline { 3 - 6 } & & $\mathbf{M} / \mathbf{M} / \mathbf{1}$ & $\mathbf{M} / \mathbf{M} / \mathbf{C}$ & $\mathbf{M} / \mathbf{M} / 1$ & $\mathbf{M} / \mathbf{M} / \mathbf{C}$ \\
\hline 10 & 15 & 10 & 45 & 0.0400 & 2.2 \\
\hline 20 & 33 & 20 & 100 & 0.0200 & 1.1 \\
\hline 30 & 50 & 30 & 130 & 0.0100 & 3.3 \\
\hline 40 & 65 & 40 & 200 & 0.0160 & 1.06 \\
\hline 60 & 75 & 50 & 290 & 0.0220 & 1.22 \\
\hline \multicolumn{3}{c}{ Calculation based on 10 nodes } \\
\hline
\end{tabular}

$\mathrm{Tq}=$ expected time delay/throughput

$\lambda=\mathrm{t}=$ arrival rate of request or throughput

$\mu=$ number of requests completed

$\mathrm{M} / \mathrm{M} / 1$ = single-tenant system

$\mathrm{M} / \mathrm{M} / \mathrm{C}=$ multi-tenant system

$\mathrm{N}=$ node

The performance of the proposed algorithms on the basis of predefined metrics and derived the simulation values can be seen in Table 2. Throughput is the first significant metric used to calculate the number of works whose jobs have been finalised. The result of the throughput should improve the performance of the designing structure. For simulation purposes, we have selected jobs with 5-10 nodes. In Table 2, column 1 describes the request rate from the client, column 2 describes the number of requests/tasks completed by the server, column 3 describes the single-tenant throughput rate, column 4 describes the multi-tenant throughput rate, column 5 shows the single-tenant time delay and column 6 
shows the multi-tenant time delay. Next, while implementing a load-balancing algorithm, overhead is involved, which should be minimised in order to work efficiently. Time of movement of the job from the master to the slave system and arrival time for results are other significant metrics. Processing time is the overhead, which should be minimised but cannot be eliminated. The amount of time taken to respond by a particular load-balancing algorithm in a distributed system is the time delay. The ability of an algorithm to perform load balancing for a system with a finite number of nodes is scalability. Finally, overall performance metrics are used to check the efficiency of the implementation at a reasonable cost.

Figure 4 shows the delay against the arrival time of single nodes or single-tenant performance assessed above. According to the simulation result, the delay times only achieved the maximum level in metrics 30 . The average performance of other numbers does not vary much when the system configuration parameters are kept constant.

Representation of data for Table 2 column 6 multi-tenant nodes in Figure 5 shows that connections with 10 nodes and five applications perform reasonably well compared to shared digital resources. Based on the simulation results, the arrival time above 30 shows a different level of delay compared to other arrival rates. This could be because of the application process at the data layer when retrieving records belonging to a tenant or a context switch between tenants in the shared resource. However, other arrival rates produced average levels of results.

In Table 2 column 4, we describe how multi-tenant throughput data provide in. Figure 6 indicates that shared digital resources assigned to achieve maximum level throughput against the arrival rate only show slight variations at throughput level 30 . Our proposed model of multi-tenancy with load balancing produced a significant level of data transactions between cloud data storage and the digital user.

\section{Benefits Of The Proposed Scheme}

As academic institutions grow larger, the structuring of the reference department becomes an issue. The reference collection should be kept in a private cloud, with all the library data distributed into a number of subject-specialised departmental libraries. The private cloud allows easy monitoring and management of cloud resources.

There are several reasons why an organisation may need to develop private cloud services. These include security concerns involving data privacy and trust, optimising existing in-house resources, the considerable cost to transfer data from local IT infrastructure to the public cloud, full control over mission-critical activities that operate behind their firewalls, and teaching and research.

Implementing private cloud computing allows digital libraries to create personalised portals for staff and students as well as for academic and administrative use by the library department. This proposed approach allows individual departments to upload data from the departmental server to centralised cloud storage. Here we discuss some of the key benefits of the proposed architecture. 


\subsection{Ease of Access to Digital Resources}

Cloud storage based on representational state transfer application programming interfaces provides web support architecture. It supports a cloud administrator and traditional block- and file-based data. As a result, it will help students and faculty members to have easy access to data in major storage locations.

\subsection{Efficient Resource Administration}

The movement from virtual data centres to private clouds necessitates the management of service consumption from managing infrastructures. Administrative capabilities can be efficiently scaled up to respond quickly and fulfil on-demand service requests by staff and students. A private cloud solution is designed to support various work environments with numerous administrative, security and service-level requirements.

\subsection{Cost-Effectiveness}

The successful implementation of private clouds not only reduces operational costs but also allows organisations to improve their quality of service, achieve faster delivery models and quickly respond to business needs. One of the highest operational costs of a virtual data centre is administrative support, and manually responding to increased demand in a cloud model will provide efficient cloud storage administration for the entire sector. Cloud computing also uses fewer physical resources and requires minimal hardware to power and maintain the service.

\subsection{Large Storage of Digital Data}

The cloud offers powerful storage and computing. It also supports a number of convenient and quick applications for powerful computing and storage of a large amount of data. Data services on cloud environments are always available and durable and can store data from a few bytes to a large number of bytes. They allow on-demand resource allocation, 24/7 accessible service and real-time configuration facilities.

\subsection{Easy Searching}

Storage of data on the cloud means that the virtual data source is stored and managed from multiple connected and distributed resources. Using cloud computing technology allows archival and disaster recovery, provides strong data backup protection, greater accessibility and reliability.

\subsection{Enhanced Data}

Access to private cloud storage requires the continuous availability of data at any time or place. A cloud provides the flexibility to accommodate decreasing or increasing demand requirements. We can increase server capacity according to the requirements of the university administration and students. The benefit of increasing server availability is that if one server goes down, user data is automatically transferred to another. 
The cloud has proven itself to be an effective solution for staff, students, administrators and the public. 'Cloud literacy' can advance and facilitate opportunities for students and staff. Providing opportunities and awareness will improve economic prospects and offer great value to students, staff, administrators, universities and colleges. Knowledge as a service is an emerging concept that integrates data and the student community. It ensures that services will be delivered to the right community of practice at the right time and in a suitable manner.

\subsection{Security}

Cloud computing presents strong security considerations. Control over the infrastructure and data security is part of private cloud installations. Cloud computing is extremely secure, and within the private cloud, user data is available behind a dedicated firewall. Public and hybrid cloud systems are often not as good as private cloud security, as to solve security issues cloud administrators are able to allocate resources.

\subsection{Development of a Professional Community}

Cloud computing technology allows libraries to build networks among library and information science professionals and other interested individuals, including those searching for information through social networking tools. Facebook and Twitter, the most famous social networking sites, play a vital role in building community power. Cooperative efforts by libraries will increase efficiency and save time.

\subsection{Digital Automation}

Many cloud providers organise different cloud-based services, such as digital content, cataloguing, acquisitions and processing systems, and include cutting-edge technologies in educational institution and digital libraries, and they also support numerous standards, such as Z39.50, XML and MARC21.

\section{Limitations}

Despite the need for multi-tenant resource sharing in the current digital world, it has certain limitations. For example, single tenants can rely on a central cloud provider, so they can provide better data for resource sharing than multi-tenant. A single node failure would disconnect the entire cloud community. Data sharing with a single tenant is safer and more secure than cloud-based resource sharing with a multi-tenant community cloud. Community clouds are more expensive than public clouds. Sharing responsibilities among organisations can be challenging.

\section{Conclusion}

Cloud computing will impact storage of and access to academic materials in the future. NIST recognises four application and four deployment models for cloud computing. Cloud libraries provide different 
applications, such as digital libraries, automated libraries, content searching, file storage and professional community building. The libraries provide the advantage of cost-effectiveness, a low investment with reduced risk, unlimited expandable storage and flexibility in access to the material. Cloud computing technology, in general, suffers from challenges relating to data confidentiality, security and access. The technology is undergoing a gradual transformation, and addressing the challenges will make it useful in academic resource sharing.

The general implications of cloud-based applications for libraries in effectively improving their services have been discussed in this study. We have highlighted the methods, techniques, procedures and benefits of storing and retrieving data efficiently from a private cloud storage system, as well as its relevance and benefits for research. Even though cloud computing provides unlimited library resources and an internetbased service that can be integrated as a whole, forming a new type of data service system faces a slew of technical, adaptive and administrative issues in reality.

Multi-tenant applications are currently supported by Salesforce, HubSpot and Netflix. In the future, they will be implemented to integrate many public and private cloud resources into the whole community. Multi-tenant applications can be organised in this way: each tenant can be assigned freely within each request, all tenant-dependent models can be assigned to a tenant, and each tenant's attributes can be unique within the community.

\section{References}

[1] S. Al-Maliki and F. Alfifi, "Using Security Features for Cloud Computing Based on New Symmetric Key Algorithm," Journal of Cloud Computing, 2016, https://doi: 10.5171/2016.179794.

[2] R. Maggiani, "Cloud computing is changing how we communicate," in 2009 IEEE International Professional Communication Conference, Jul. 2009, pp. 1-4, https://doi: 10.1109/IPCC.2009.5208703.

[3] J. P. Srivastava and V. K. Verma, "Cloud computing in libraries: Its needs, applications, issues and best practices," in 2015 4th International Symposium on Emerging Trends and Technologies in Libraries and Information Services, Jan. 2015, pp. 33-38, https:// doi:10.1109/ETTLIS.2015.7048168.

[4] R. Sanchati and G. Kulkarni, "Cloud Computing in Digital and University Libraries," Global Journal of Computer Science and Technology, vol. 11, no. 12, pp. 36-42, 2011.

[5] D. K. Kumar, "Application of Cloud Technology in Digital Library," International Journal of Computer Science Issues (IJCSI), vol. 9, no. 3, pp. 374-378, 2012.

[6] F. Sabahi, "Cloud computing security threats and responses," in 2011 IEEE 3rd International Conference on Communication Software and Networks, May 2011, pp. 245-249, https://doi: 10.1109/ICCSN.2011.6014715. 
[7] S. Aljerewi, "The impact of a proposed technology based on cloud computing in the development of practical knowledge skills among students of the Faculty of Education Princess Nourah Bint Abdul Rahman University," IUG Journal of Educational and Psychology Sciences, vol. 26, no. 3, pp. 54-84, 2018.

[8] S. Bellasio, "5 Best Practices for a Successful Cloud Migration," 2018.

https://vmblog.com/archive/2018/05/18/5-best-practices-for-a-successful-cloudmigration.aspx\#.YgepUurP1PZ (accessed Feb. 12, 2022).

[9] J. Sen, "Security and Privacy Issues in Cloud Computing," in Architectures and Protocols for Secure Information Technology Infrastructures, vol. 3, A. Ruiz-Martinez, R. Marin-Lopez, and F. Pereniguez-Garcia, Eds. Hershey, PA: IGI Global, 2014, pp. 1-45, https://doi: 10.4018/978-1-4666-4514-1.ch001.

[10] S. Namasudra, "Cloud computing: A new era," Journal of Fundamental and Applied Sciences, vol. 10, no. 2, Art. no. 2, May 2018, https://doi: 10.4314/jfas.v10i2.9.

[11] N. Tyagi, N. Passi, and S. K. Baberwal, "Cloud Computing \& it's Applications in Library Systems," International Journal of Research in Engineering and Technology, vol. 4, pp. 45-47, 2015.

[12] M. Marayaja, "Cloud computation in library: advantage and disadvantages," International Research Journal of Management Science \& Technology, vol. 7, no. 6, pp. 117-122, 2016.

[13] NIST Cloud Computing Standards and Roadmap, "National Institute of Standards and Technology, 500-291, 2nd ser." 2013. [Online]. Available: https://nvlpubs.nist.gov/nistpubs/SpecialPublications/NIST.SP.500-291r2.pdf

[14] V. Diaconita, A.-R. Bologa, and R. Bologa, "Hadoop Oriented Smart Cities Architecture," Sensors, vol. 18, no. 4, Art. no. 4, Apr. 2018, https://doi: 10.3390/s18041181.

[15] V. Inukollu, S. Arsi, and S. Ravuri, "Security Issues Associated with Big Data in Cloud Computing," International Journal of Network Security \& Its Applications, vol. 6, no. 3, pp. 45-56, May 2014, https://doi: 10.5121/ijnsa.2014.6304.

[16] R. Arokia, A. Paul Rajan, and S. Shanmugapriyaa, "Evolution of Cloud Storage as Cloud Computing Infrastructure Service," IOSR, vol. 1, pp. 2278-661, Jun. 2012, https://doi: 10.9790/0661-0113845.

[17] C. Thota, G. Manogaran, D. Lopez, and R. Sundarasekar, "Architecture for Big Data Storage in Different Cloud Deployment Models," in Research Anthology on Architectures, Frameworks, and Integration Strategies for Distributed and Cloud Computing, IGI Global, 2021, pp. 178-208, https:// doi: 10.4018/978-1-7998-5339-8.ch009.

[18] M. Bengtsson, "How to plan and perform a qualitative study using content analysis," NursingP/us Open, vol. 2, pp. 8-14, Jan. 2016, https://doi: 10.1016/j.npls.2016.01.001. 
[19] ZNetlive, "What is load balancing in Cloud Computing and what are its advantages?," ZNetLive Blog A Guide to Domains, Web Hosting \& Cloud Computing, 2017, https://www.znetlive.com/blog/what-isload-balancing-in-cloud-computing-and-its-advantages/ (accessed Feb. 12, 2022).

[20] H. Yaish, M. Goyal, and G. Feuerlicht, "A novel multi-tenant architecture design for software as a service application," in CLOSER 2012 - Proceedings of the 2nd International Conference on Cloud Computing and Services Science, Jan. 2012, pp. 82-88.

\section{Declarations}

\section{Ethical approval}

Not applicable.

\section{Consent to participate}

Not applicable.

\section{Consent for publication}

Not applicable.

\section{Availability of data and materials}

The datasets used and analyzed during the current study are available from the corresponding author on reasonable request.

\section{Competing Interest}

The author declares no competing interest.

\section{Funding}

This research is not funded by any resource.

\section{Acknowledgement}

The author is very thankful to all the associated personnel in any reference that contributed in/for the purpose of this research.

\section{Figures}



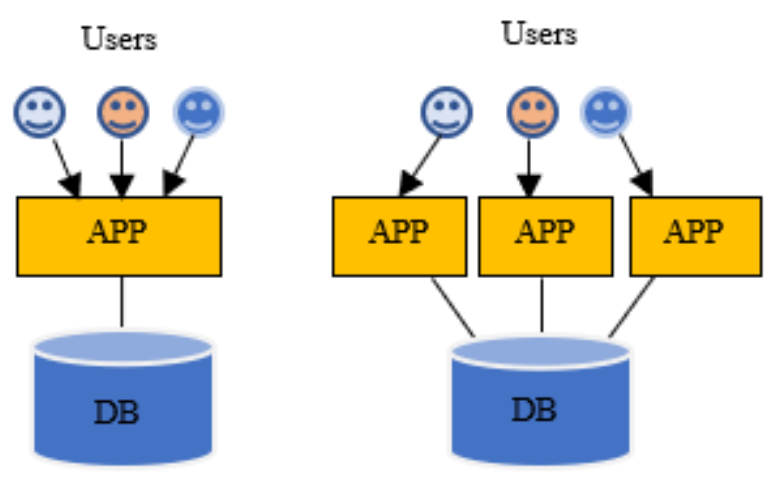

Figure 1

Single-tenant and multi-tenant modules

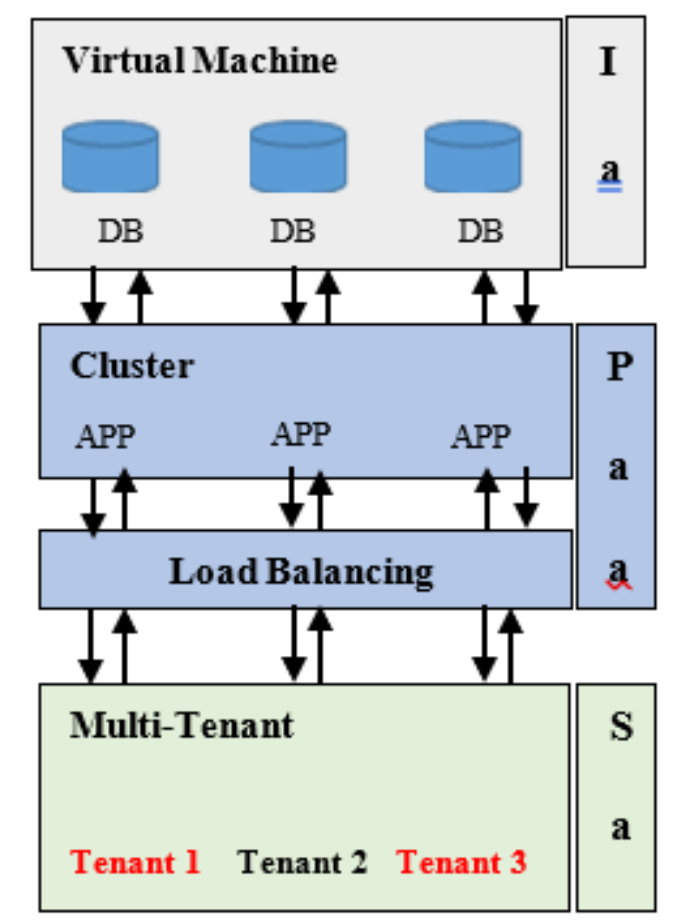

Figure 2

Structure of digital resource sharing using multi-tenancy 


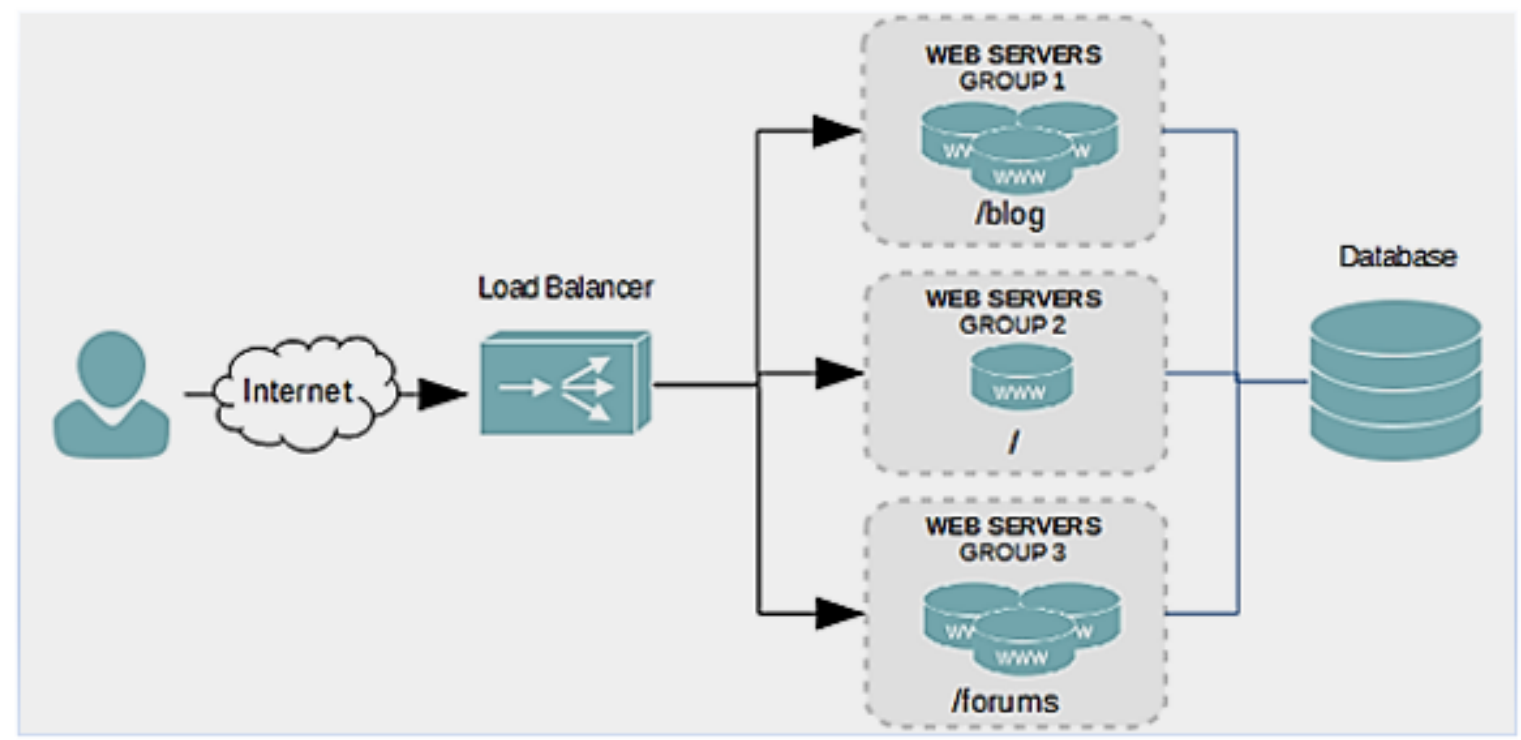

Figure 3

Cloud-based data sharing using load balancing [19]

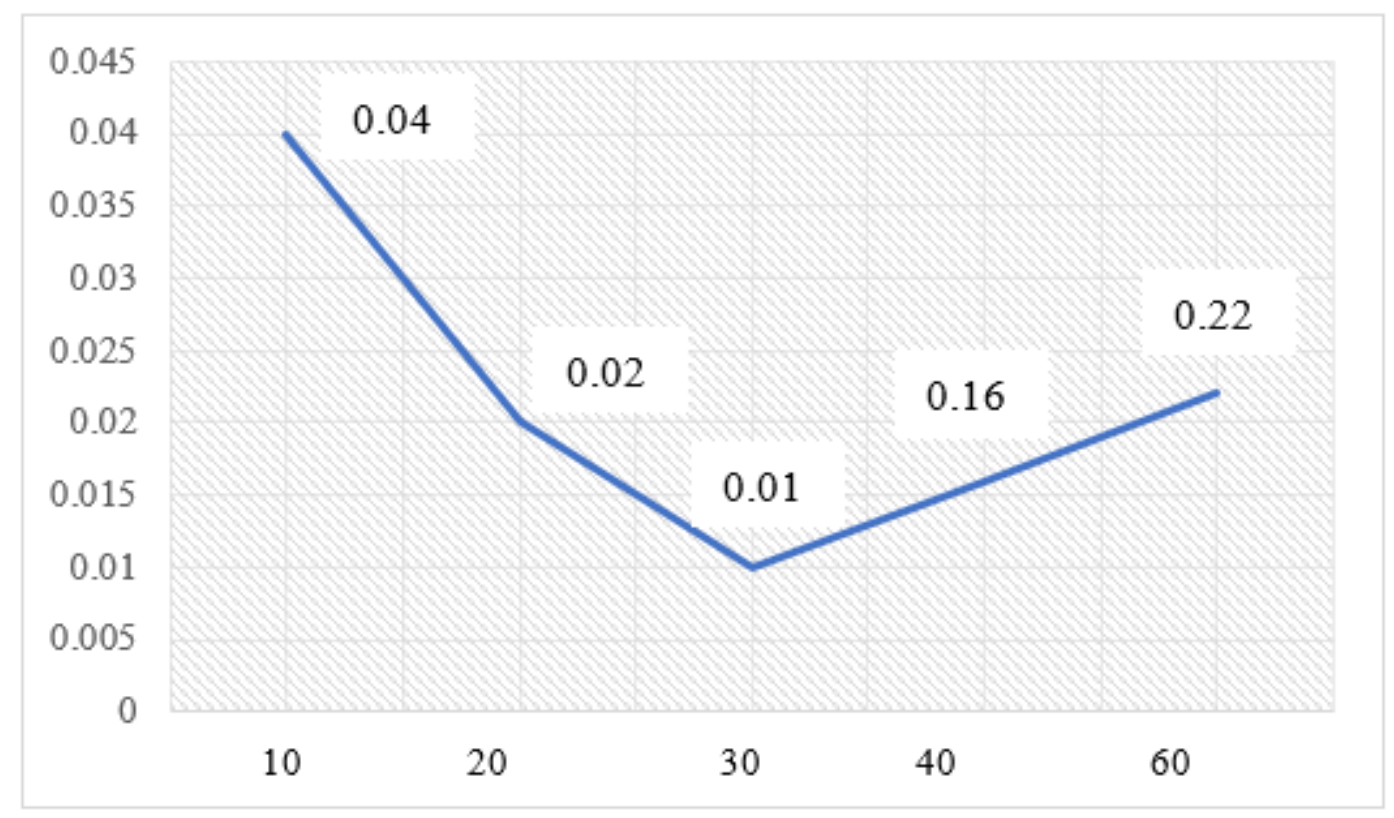

Figure 4

$\mathrm{M} / \mathrm{M} / 1$ system delay against the arrival time 


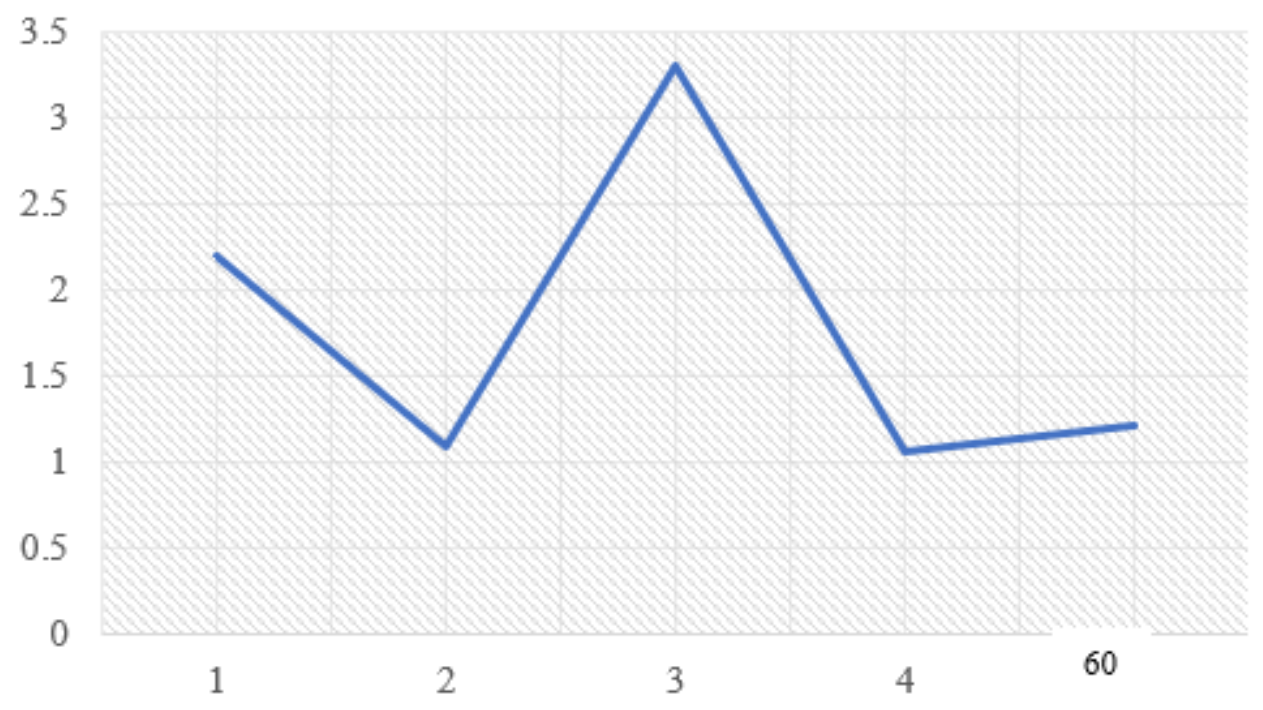

Figure 5

$M / M / C$ system delay against the arrival time

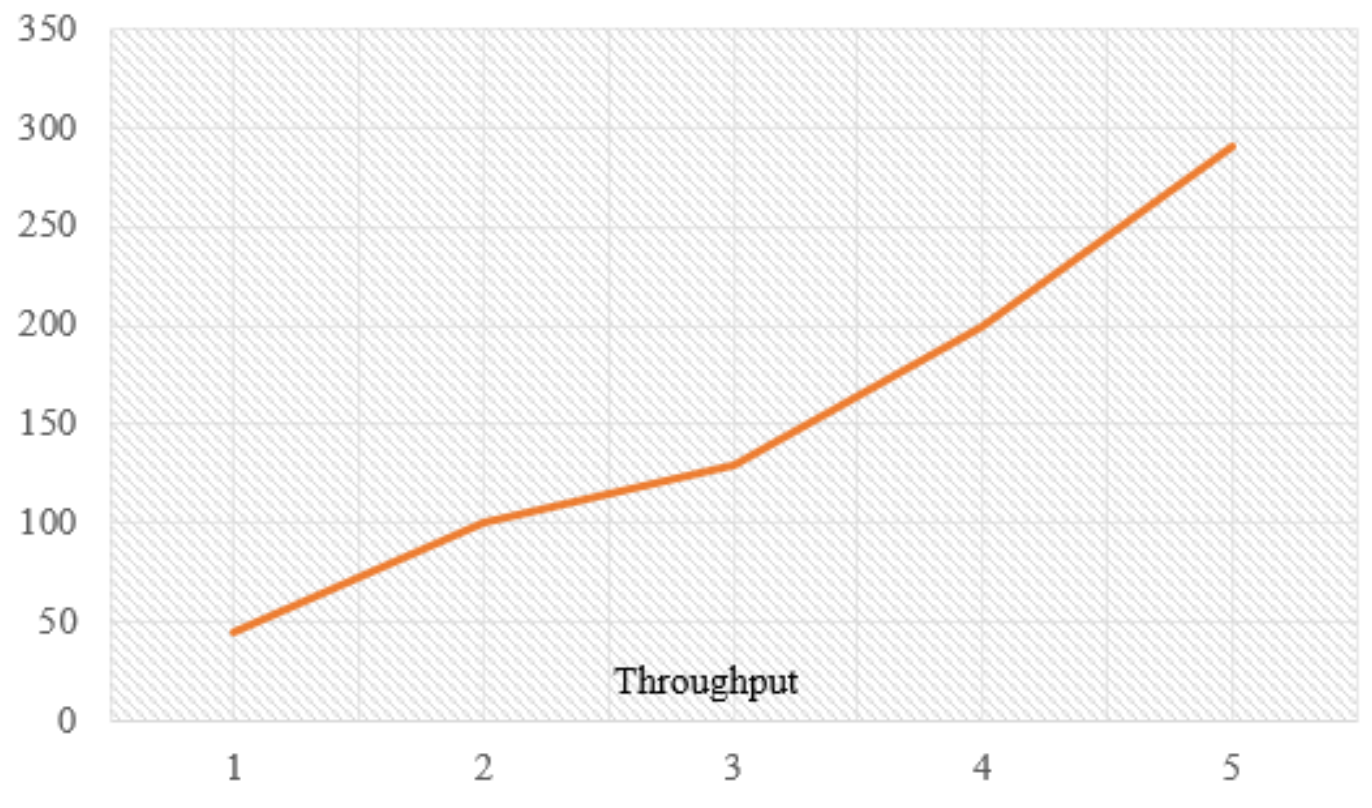

Figure 6

Arrival rate throughput of $\mathrm{M} / \mathrm{M} / \mathrm{C}$ 